\title{
More Anti-Chain Based Refinement Checking
}

\author{
Ting Wang ${ }^{1}$, Songzheng Song ${ }^{2}$, Jun $\mathrm{Sun}^{3}$, Yang $\mathrm{Liu}^{2}$, Jin Song Dong ${ }^{2}$, Xinyu Wang ${ }^{1}$ \\ and Shanping $\mathrm{Li}^{1}$ \\ ${ }^{1}$ College of Computer Science and Technology, Zhejiang University \\ $\{$ qdw, wangxinyu, shan $\}$ e z ju. edu.cn \\ 2 National University of Singapore \\ \{songsongzheng@, tslliuya@, dongjs@comp.\}nus.edu.sg \\ 3 Singapore University of Technology and Design \\ sunjunesutd.edu.sg
}

\begin{abstract}
Refinement checking plays an important role in system verification. It establishes properties of an implementation by showing a refinement relationship between the implementation and a specification. Recently, it has been shown that anti-chain based approaches increase the efficiency of trace refinement checking significantly. In this work, we study the problem of adopting anti-chain for stable failures refinement checking, failures-divergence refinement checking and probabilistic refine checking (i.e., a probabilistic implementation against a nonprobabilistic specification). We show that the first two problems can be significantly improved, because the state space of the product model may be reduced dramatically. Though applying anti-chain for probabilistic refinement checking is more complicated, we manage to show improvements in some cases. We have integrated these techniques into the PAT model checking framework. Experiments are conducted to demonstrate the efficiency of our approach.
\end{abstract}

\section{Introduction}

Model checking has established itself as an effective technique for system verification. It works by exhaustively searching through the state space in order to show that an implementation model, in certain modeling language, satisfies a property. Properties are often specified using temporal logic formulae such as CTL or LTL, in other words, a language different from the modeling language. An alternative approach is called refinement checking. Different from temporal-logic based model checking, refinement checking shows a refinement relationship between two models in the same language, one modeling an implementation and one modeling a specification. If the specification satisfies certain property and the refinement relationship is strong enough to preserve the property, we imply that the property is satisfied by the implementation. A variety of refinement relationships have been defined, which preserve different classes of properties. For instance, safety can be verified by showing a trace refinement relationship. Combination of safety and liveness is verified by showing a stable failures refinement relationship if the system is divergence-free or otherwise by showing a failuresdivergence refinement relationship. The readers are refer to [12] for a discussion on the expressiveness of different refinement. 
Refinement checking has been traditionally used to verify CSP [11]. The success of the FDR refinement checker [1], which supports fully automatic checking of the abovementioned refinement relationships, evidences the usefulness of refinement checking. Recently, Sun et al. extended the idea of automated trace refinement checking to probabilistic systems [14], which we refer to as probabilistic refinement checking in this work ${ }^{4}$. The idea is that, given a probabilistic implementation model (which has the semantics of a Markov Decision Process) and a non-probabilistic specification model, probabilistic refinement checking calculates the probability of the implementation exhibiting traces of the specification model. This is useful as, for instance, if the specification model captures desired system behaviors, the result is the probability of the implementation behaving 'well'.

Due to the non-determinism in the specification, refinement checking often relies on the classic subset construction approach. The subset construction is used to build a deterministic finite-state automaton (DFA) from the specification, which is in general a non-deterministic finite-state automaton (NFA). Next, refinement checking works by computing the synchronous product of the implementation and transforming the problem into a reachability analysis problem in the product. In the worst case, the resultant DFA could have exponentially more states than the original NFA. As a result, refinement checking suffers from state space explosion. Recently, Wulf et al. proposed an approach (for solving the language universality problem and trace refinement checking) named anti-chain [16]. It has been shown that anti-chain based trace refinement checking outperforms the previous approaches significantly. The key point of anti-chain based approaches is that the complete subset construction and computing the complete state space of the product are avoided. Given that the existing approaches for checking other refinement relationships are all based on the subset construction, it is only naturally to investigate whether anti-chain can be used for better performance as it did for trace refinement checking.

In this work, we study three kinds of refinement checking, in particular, stable failures refinement, failures-divergence refinement and probabilistic refinement checking. The problem is non-trivial as we need to formally prove that anti-chain works with stable failures semantics and failures-divergence semantics. Furthermore, it is complicated for probabilistic refinement checking as omitting parts of the product would affect the probability. We make the following technical contribution. Firstly, we show that antichain can be readily used to improve stable failures refinement and failures-divergence refinement. Secondly, we show that anti-chain can be used to improve probabilistic refinement checking in some particular cases, using an iterative probability calculation method. Lastly, we implement the technique in the PAT model checker [13] and show improvement over existing approaches (significant for stable failures refinement and failures-divergence refinement).

Related works This work is related to research on anti-chain based model checking. Wulf et al. proposed the anti-chain based approach for checking the language universality and trace refinement of NFA [16]. It has been shown that the anti-chain based approach may outperform the standard ones by several orders of magnitude. Their fol-

\footnotetext{
${ }^{4}$ Probabilistic refinement has been used by different researchers to mean different things.
} 
lowing works show that significant improvements can also be brought to the model checking problem of LTL by using anti-chain based algorithms [8,17]. Later Abdulla et al. improved the approach through exploiting a simulation relation on the states of NFA [2]. Remotely related are anti-chain based methods for solving other problems, e.g., the LTL realizability and synthesis problem $[7,10]$ and the universality and language inclusion problem of non-deterministic tree automata (NTA) [2,6]. In our work, we focus on stable failures refinement, failures-divergence refinement and probabilistic refinement checking.

Organization The remainder of the paper is organized as follows. Section 2 reviews trace refinement and anti-chain based trace refinement checking. Section 3 presents algorithms for anti-chain based stable failures refinement checking and failures-divergence refinement checking. Section 4 shows that anti-chain can be used to improve probabilistic refinement checking (with a non-probabilistic specification model). Lastly, Section 5 concludes the paper.

\section{Background}

In this section, we review previous work on anti-chain based trace refinement checking.

\subsection{Trace Refinement}

Let $\Sigma$ be a set of event names; $\tau$ denote an invisible event; and $\Sigma_{\tau}$ denote $\Sigma \cup\{\tau\}$.

Definition 1 (LTS). A labeled transition system (LTS) is a tuple $\mathcal{L}=(S$, init, Act, $T)$ where $S$ is a set of states; init $\in S$ is an initial state; Act $\subseteq \Sigma_{\tau}$ is a set of events and $T: S \times$ Act $\times S$ is a labeled transition relation.

For simplicity, $\left(s, e, s^{\prime}\right) \in T$ is sometimes written as $s \stackrel{e}{\rightarrow} s^{\prime}$. An LTS is deterministic if and only if for all $s \in S$ and $s \in \Sigma_{\tau}$, if $s \stackrel{e}{\rightarrow} u$ and $s \stackrel{e}{\rightarrow} v$, then $u=v$. We write enable $(s)$ to denote the set $\left\{e \mid \exists s^{\prime} . s \stackrel{e}{\rightarrow} s^{\prime}\right\}$. We write $u \rightsquigarrow v$ if there exists a finite sequence of states $\left\langle s_{0}, s_{1}, \cdots, s_{n}\right\rangle$ such that $s_{i} \stackrel{\tau}{\rightarrow} s_{i+1}$ for all $i$ and $u=s_{0}$ and $v=s_{n}$. We write $u \stackrel{e}{\rightsquigarrow} v$ if $u \rightsquigarrow u^{\prime}$ and $u^{\prime} \stackrel{e}{\rightarrow} v^{\prime}$ and $v^{\prime} \rightsquigarrow v^{\prime}$. A finite sequence of events $\left\langle e_{0}, e_{1}, \cdots, e_{n}\right\rangle$ is a trace of $\mathcal{L}$ if and only if there exists a sequence of state $\left\langle s_{0}, s_{1}, \cdots, s_{n}\right\rangle$ such that $s_{i} \stackrel{e_{i}}{\rightsquigarrow} s_{i+1}$ for all $i$ and $s_{0}=i n i t$. The traces of $\mathcal{L}$ are denoted as $\operatorname{traces}(\mathcal{L})$.

Definition 2 (LTS Synchronous Product). Let $\mathcal{L}_{i}=\left(S_{i}\right.$, init $_{i}$, Act $\left._{i}, T_{i}\right)$ where $i \in$ $\{1,2\}$ be two LTSs such that $\tau \notin A$ ct $t_{2}$. The synchronous product of $\mathcal{L}_{1}$ and $\mathcal{L}_{2}$, written as $\mathcal{L}_{1} \times \mathcal{L}_{2}$, is an LTS $\mathcal{L}=(S$, init, Act, $T)$ such that $S=S_{1} \times S_{2}$; init = $\left.\left(\text { init }_{1} \text {, init }\right)_{2}\right) ; A c t=A c t_{1} \cup$ Act $_{2}$; and $T$ is the minimum labeled transition relation satisfying the following conditions.

- If $\left(s_{1}, \tau, s_{1}^{\prime}\right) \in T_{1},\left(\left(s_{1}, s_{2}\right), \tau,\left(s_{1}^{\prime}, s_{2}\right)\right) \in T$ for all $s_{2} \in S_{2}$;

- If $\left(s_{1}, e, s_{1}^{\prime}\right) \in T_{1}$ and $\left(s_{2}, e, s_{2}^{\prime}\right) \in T_{2}$ and $e \notin \tau,\left(\left(s_{1}, s_{2}\right), e,\left(s_{1}^{\prime}, s_{2}^{\prime}\right)\right) \in T$.

Notice that all events except $\tau$ are to be synchronized by the two LTSs. 
Definition 3 (Trace Refinement). Let $\mathcal{L}_{i}$ where $i \in\{1,2\}$ be two LTSs. $\mathcal{L}_{1}$ tracerefines $\mathcal{L}_{2}$ if and only if traces $\left(\mathcal{L}_{1}\right) \subseteq \operatorname{traces}\left(\mathcal{L}_{2}\right)$.

The standard approach for trace refinement is based on the subset construction. That is, the specification $L T S_{2}$ is transformed into trace-equivalent deterministic LTS without $\tau$-transitions through the process of determinization. Let $\mathcal{L}=(S$, init, Act, $T)$ be an LTS. The determinized LTS of $\mathcal{L}$ is $\operatorname{det}(\mathcal{L})=\left(S^{\prime}\right.$, init $\left.^{\prime}, A c t^{\prime}, T^{\prime}\right)$ where $S^{\prime} \subseteq 2^{S}$ is a set of sets of states, init ${ }^{\prime}=\{s \mid$ init $\rightsquigarrow s\} ; A c t^{\prime}=A c t \backslash\{\tau\}$ and $T^{\prime}$ is a transition relation satisfying the following condition: $\left(N, e, N^{\prime}\right) \in T^{\prime}$ if and only if $N^{\prime}=\left\{s^{\prime} \mid\right.$ $\left.\exists s: N . s \stackrel{e}{\rightsquigarrow} s^{\prime}\right\}$. Notice that states which can be reached via the same trace are grouped together in $\operatorname{det}(\mathcal{L})$.

Given an implementation $\mathcal{L}_{1}$ and a specification $\mathcal{L}_{2}$, the standard trace refinement checking is to construct (often on-the-fly) the product $\mathcal{L}_{1} \times \operatorname{det}\left(\mathcal{L}_{2}\right)$ and then search for a state of the product $\left(s_{1}, s_{2}\right)$ (where $s_{1}$ is a state of $\mathcal{L}_{1}$ and $s_{2}$ is a set of states in $\mathcal{L}_{2}$ ) such that $s_{2}$ is an empty set. Such a state is called a TR-witness state. In the worst case, this algorithm has a complexity exponential in the number of states of $\mathcal{L}_{2}$.

\subsection{Trace Refinement Checking with Anti-Chain}

It has been shown that trace refinement checking based on anti-chain offers significantly better performance [16]. Given two LTSs $\mathcal{L}_{1}$ and $\mathcal{L}_{2}$, the anti-chain method explores a 'simulation' relation in $\mathcal{L}_{1} \times \operatorname{det}\left(\mathcal{L}_{2}\right)$. Given any two states $\left(s_{1}, s_{2}\right)$ and $\left(s_{1}^{\prime}, s_{2}^{\prime}\right)$ of $\mathcal{L}_{1} \times \operatorname{det}\left(\mathcal{L}_{2}\right)$, let $\left(s_{1}^{\prime}, s_{2}^{\prime}\right) \leq\left(s_{1}, s_{2}\right)$ denote $s_{1}=s_{1}^{\prime}$ and $s_{2} \subseteq s_{2}^{\prime}$.

Proposition 1. If $\left(s_{1}^{\prime}, s_{2}^{\prime}\right) \leq\left(s_{1}, s_{2}\right)$ and $\left(s_{1}, s_{2}\right) \stackrel{e}{\rightarrow}(u, v)$, then there exists $\left(u^{\prime}, v^{\prime}\right)$ such that $\left(s_{1}^{\prime}, s_{2}^{\prime}\right) \stackrel{e}{\rightarrow}\left(u^{\prime}, v^{\prime}\right)$ and $u^{\prime}=u$ and $v \subseteq v^{\prime}$.

By the above proposition, it can be readily shown that a TR-witness state is reachable from $\left(s_{1}^{\prime}, s_{2}^{\prime}\right)$ implies that a TR-witness state must be reachable from $\left(s_{1}, s_{2}\right)$. As a result, if $\left(s_{1}, s_{2}\right)$ has been explored, we can skip $\left(s_{1}^{\prime}, s_{2}^{\prime}\right)$.

Formally, an anti-chain is a set $A$ of sets such that $x \nsubseteq y$ and $y \nsubseteq x$ for all $x \in A$ and $y \in A$, i.e., any pair of sets in $A$ are incomparable. An anti-chain supports two operations. One is to check whether it contains a subset of a given set. let $x$ be the given set, we denote $x \Subset A$ if and only if there exists $y \in A$ such that $y \subseteq x$. The other is to add a given set $x$ in $A$. $A \uplus x$ is defined as $\{y \mid y \in A \wedge x \nsubseteq y\} \cup\{x\}$, i.e., $A$ ש $x$ contains $x$ and all sets in $A$ which is not a superset of $x$. Obviously, an empty set is an anti-chain by definition.

Algorithm 1 shows the anti-chain based algorithm. In an abuse of notation, we write $(s, X) \Subset A$ to denote that the set $(\{s\} \cup X) \Subset A$; and $A \uplus(s, X)$ to denote $A \uplus(\{s\} \cup$ $X)$. The algorithm works as follows. After initializing the stack working and the set antichain, the algorithm pops one state $(\mathrm{impl}, \mathrm{spec})$ from working and adds it to the set antichain, and then generates all successors of the state and adds them to working unless $\left(\mathrm{impl}^{\prime}\right.$, spec $\left.^{\prime}\right) \Subset$ antichain is true, till the stack working is empty or a TRwitness state is found. We remark that antichain keeps to be an anti-chain during this algorithm, because line 5 and line 13 guarantee there are no subsets or supersets of the new added state in the updated antichain. Soundness of the algorithm can be referred to in [2] [16]. 

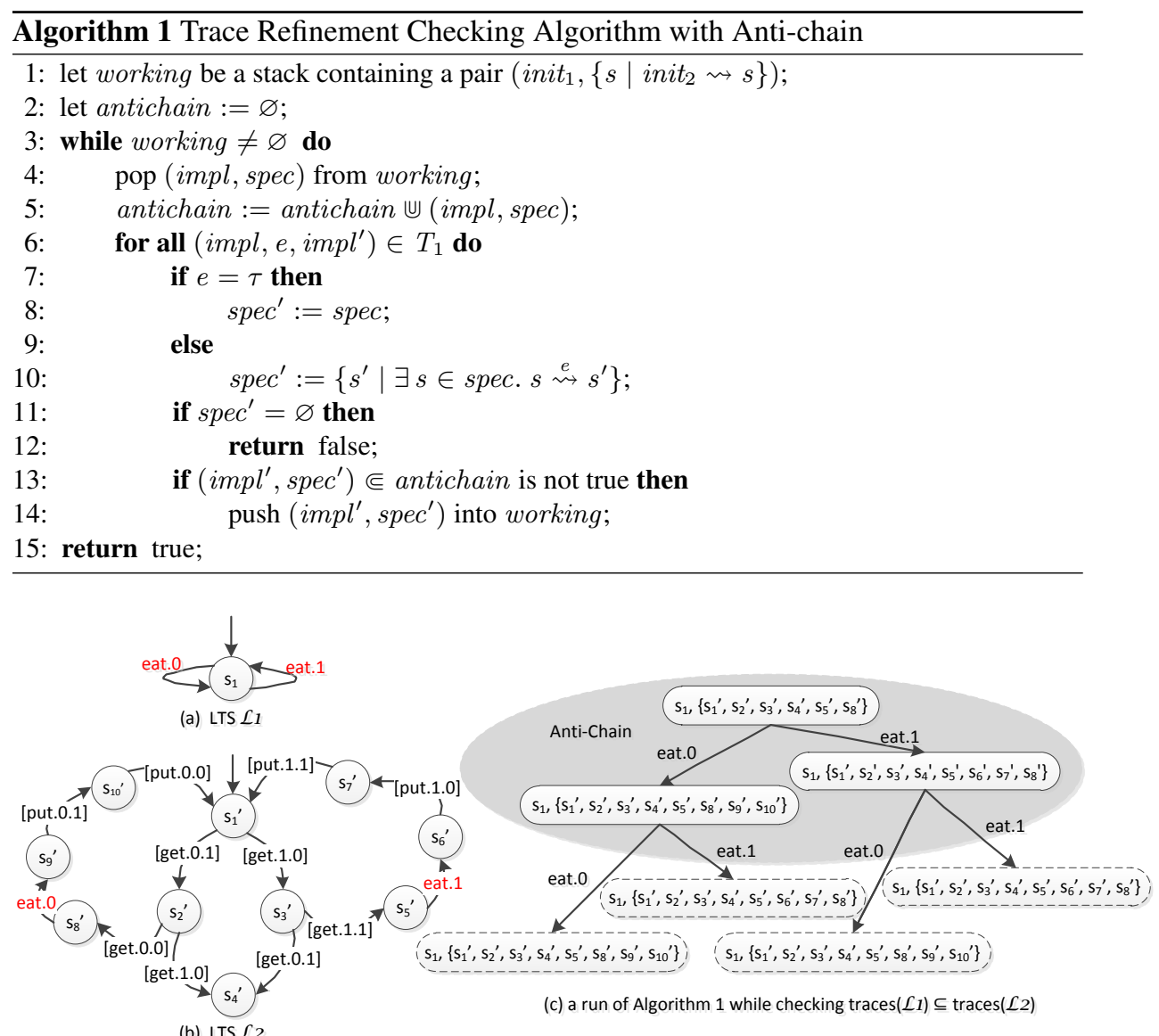

Fig. 1. Trace Refinement Checking Algorithm with Anti-Chain

Theorem 1. [16] Algorithm 1 returns true if and only if traces $\left(\mathcal{L}_{1}\right) \subseteq \operatorname{traces}\left(\mathcal{L}_{2}\right)$.

Example 1. Figure 1 shows a simple example of dining philosopher [11] to demonstrate how Algorithm 1 works. The problem is summarized as $N$ philosophers sitting around a round table with a single fork between each pair, and each philosopher requiring both neighboring forks to eat. The LTS $\mathcal{L}_{2}$ in Figure 1 (b) shows the complete state graph of the system with two philosophers. The invisible events is denoted as [event], i.e., [get.0.1] means that the hidden event is philosopher 0 getting the right fork which is represented as 1 . Note that checking $\operatorname{traces}\left(\mathcal{L}_{1}\right) \subseteq \operatorname{traces}\left(\mathcal{L}_{2}\right)$ is to confirm whether every philosopher can eat. From Figure 1 (c) we can see that the search does not continue from the state $\left(s_{1},\left\{s_{1}^{\prime}, s_{2}^{\prime}, s_{3}^{\prime}, s_{4}^{\prime}, s_{5}^{\prime}, s_{8}^{\prime}, s_{9}^{\prime}, s_{10}^{\prime}\right\}\right)$ because $\left\{s_{1}^{\prime}, s_{2}^{\prime}, s_{3}^{\prime}, s_{4}^{\prime}, s_{5}^{\prime}, s_{8}^{\prime}\right\} \subseteq\left\{s_{1}^{\prime}, s_{2}^{\prime}, s_{3}^{\prime}, s_{4}^{\prime}, s_{5}^{\prime}, s_{8}^{\prime}, s_{9}^{\prime}, s_{10}^{\prime}\right\}$. In this case, Algorithm 1 generates 3 states which are labeled with Anti-Chain, while the classical algorithm based on subset construction generates 7 states. 


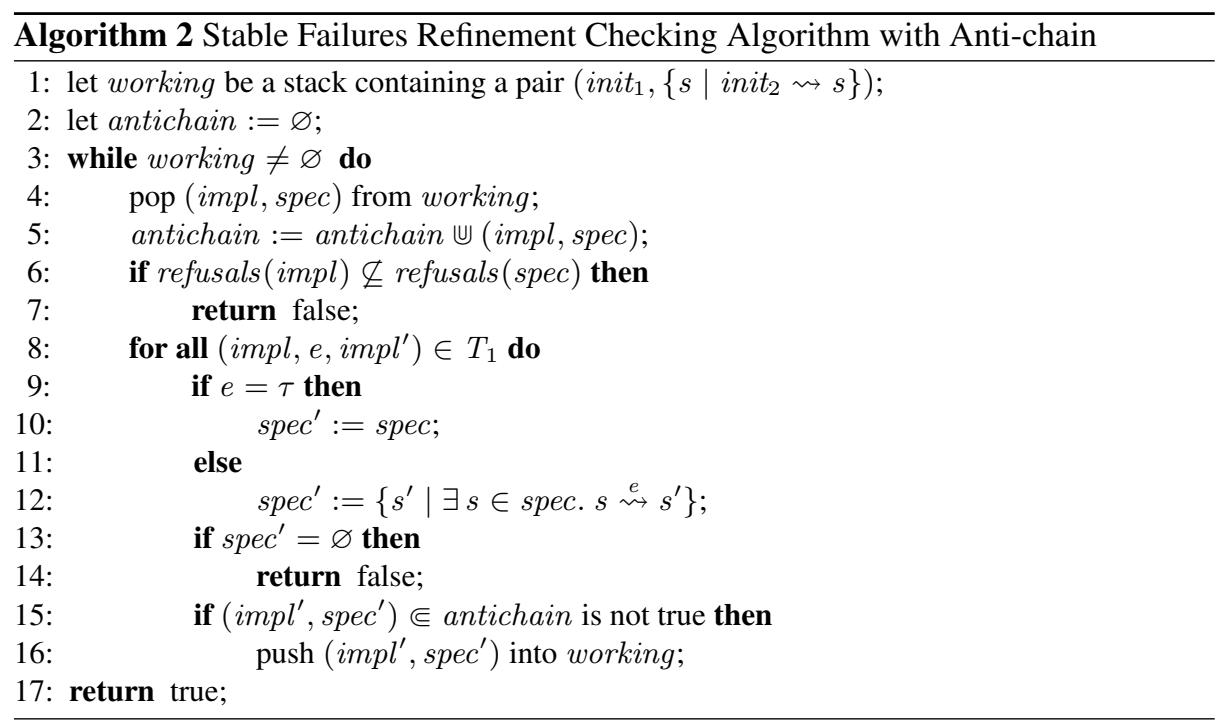

\section{Failures/Divergence Refinement Checking with Anti-Chain}

In this section, we demonstrate that anti-chain can be used to improve stable failures refinement checking and failures-divergence refinement checking.

\subsection{Stable Failures Refinement Checking}

Let $\mathcal{L}=(S$, init, Act, $T)$ be an LTS. Given a state $s \in S, s$ is stable if $\tau \notin$ enable $(s)$. Given a stable state $s$, the refusals of $s$, written as refusals $(s)$, is defined as $\{X \mid$ $\exists s^{\prime} . s \rightsquigarrow s^{\prime} \wedge \tau \notin$ enable $\left(s^{\prime}\right) \wedge X \subseteq \Sigma \backslash$ enable $\left.\left(s^{\prime}\right)\right\}$. The failures of $\mathcal{L}$, written as failures $(\mathcal{L})$, is defined as $\left\{(\operatorname{tr}, X): \Sigma^{*} \times 2^{\Sigma} \mid \exists s\right.$. init $\left.\stackrel{t r}{\rightsquigarrow} s \wedge X \in \operatorname{refusals}(s)\right\}$ where init $\stackrel{t r}{\leftrightarrow} s$ denotes that there exists a run $\left\langle s_{0}, e_{0}, s_{1}, e_{1}, \cdots, e_{n}, s_{n+1}\right\rangle$ such that $s_{0}=$ init and $s_{n+1}=s$ and $\operatorname{tr}=\left\langle e_{0}, e_{1}, \cdots, e_{n}\right\rangle$.

Definition 4 (Stable Failures Refinement). Let $\mathcal{L}_{i}$ where $i \in\{1,2\}$ be two LTSs. $\mathcal{L}_{1}$ refines $\mathcal{L}_{2}$ in stable failures semantics if and only if failures $\left(\mathcal{L}_{1}\right) \subseteq$ failures $\left(\mathcal{L}_{2}\right)$.

The existing stable failures refinement checking algorithm [1] works by searching for a state $(x, y)$ of $\mathcal{L}_{1} \times \operatorname{det}\left(\mathcal{L}_{2}\right)$ such that $y=\varnothing$ or refusals $(x) \nsubseteq$ refusals $(y)$. Such a state is called a SFR-witness state. In the following, we extend Algorithm 1 for stable failures refinement checking. Given a set states $x$, we write refusals $(x)$ to denote $\{r \mid$ $\exists s \in x . r \in$ refusals $(s)\}$. The algorithm is shown in Algorithm 2.

Lemma 1. For every state $\left(s_{1}, s_{2}\right)$ of $\mathcal{L}_{1} \times \operatorname{det}\left(\mathcal{L}_{2}\right)$, for all $\left(s_{1}, s_{2}^{\prime}\right)$ in the product, if $s_{2}^{\prime} \subseteq s_{2}$, then a SFR-witness state is reachable from $\left(s_{1}, s_{2}\right)$ implies a SFR-witness state is reachable from $\left(s_{1}, s_{2}^{\prime}\right)$. 


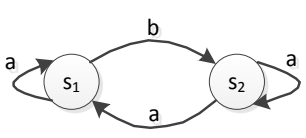

(a) LTS $\mathcal{L}_{1}$

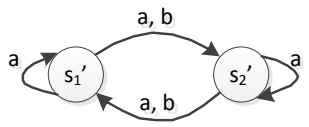

(b) LTS $\mathcal{L} 2$

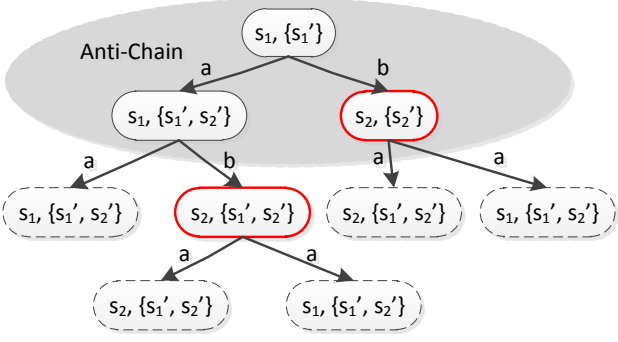

(c) a run of Algorithm 2 while checking failures $\left(\mathcal{L}_{1}\right) \subseteq$ failures $\left(\mathcal{L}_{2}\right)$

Fig. 2. Stable Failures Refinement Checking Algorithm with Anti-Chain

Proof By induction. The base case is that $\left(s_{1}, s_{2}\right)$ is a SFR-witness state, then $s_{2}=$ $\varnothing$ or refusals $\left(s_{1}\right) \nsubseteq$ refusals $\left(s_{2}\right)$. Because $s_{2}^{\prime} \subseteq s_{2}$ by assumption, refusals $\left(s_{2}^{\prime}\right) \subseteq$ refusals $\left(s_{2}\right)$. Then we have $s_{2}^{\prime}=\varnothing$, or refusals $\left(s_{1}\right) \not$ refusals $\left(s_{2}^{\prime}\right)$. Thus, $\left(s_{1}, s_{2}^{\prime}\right)$ is a SFR-witness state. Next, we prove the induction step. Assume that $\left(s_{1}, s_{2}\right)$ satisfies the condition, i.e., for any state $\left(s_{1}, s_{2}^{\prime}\right)$ such that $s_{2}^{\prime} \subseteq s_{2}$, a SFR-witness state is reachable from $\left(s_{1}, s_{2}\right)$ implies a SFR-witness state is reachable from $\left(s_{1}, s_{2}^{\prime}\right)$. Let $(x, y)$ be a state of the product such that $(x, y) \stackrel{e}{\rightarrow}\left(s_{1}, s_{2}\right)$. For all $\left(x, y^{\prime}\right)$ such that $y^{\prime} \subseteq y$, we can get $\left(x, y^{\prime}\right) \stackrel{e}{\rightarrow}\left(s_{1}, s_{2}^{\prime}\right)$ such that $s_{2}^{\prime} \subseteq s_{2}$. Therefore, the induction step holds by induction hypothesis. Thus, the lemma is true.

Theorem 2. Algorithm 2 returns true if and only if failures $\left(\mathcal{L}_{1}\right) \subseteq$ failures $\left(\mathcal{L}_{2}\right)$.

Proof For a state $S$ of $\mathcal{L}_{1} \times \operatorname{det}\left(\mathcal{L}_{2}\right)$, define $\operatorname{Dist}(S) \in N \cup\{\infty\}$ as the length of the shortest SFR-witness trace from $S$ (if a SFR-witness state is not reachable from $S, \operatorname{Dist}(S)=\infty)$. For a set of states States, if States $=\varnothing, \operatorname{Dist}($ States $)=\infty$, otherwise, Dist(States) $=\min _{S \in \text { States }} \operatorname{Dist}(S)$. The predicate $S F R($ States $)$ is true if and only if all the states in States are not SFR-witness states. Then the correctness of Algorithm 2 can be proved using the two invariants below. The invariants can be proved in a very similar way to [2].

1. $\neg S F R($ antichain $\cup$ working $) \Rightarrow \neg S F R\left(\left\{\left(i,\left\{s \mid\right.\right.\right.\right.$ init $\left.\left._{2} \rightsquigarrow s\right\}\right) \mid i \in$ init $\left.\left._{1}\right\}\right)$.

2. $\neg \operatorname{SFR}\left(\left\{\left(i,\left\{s \mid\right.\right.\right.\right.$ init $\left.\left._{2} \rightsquigarrow s\right\}\right) \mid i \in$ init $\left.\left._{1}\right\}\right) \Rightarrow \operatorname{Dist}($ antichain $)>\operatorname{Dist}($ working $)$.

Because the number of state is finite and all states are only visited once, Algorithm 2 eventually terminates. Algorithm 2 returns false only if the state $s^{\prime} c^{\prime}$ is an empty set on line 13 , or (impl, spec) satisfies the condition refusals $(\mathrm{impl}) \nsubseteq$ refusals (spec) on line 6. The former case has been proved in Algorithm 1. In the latter case, $(\mathrm{impl}, \mathrm{spec})$ is a SFR-witness state, and hence $S F R$ (antichain $\cup$ working) is false. By invariant 1, $\mathcal{L}_{1}$ cannot refine $\mathcal{L}_{2}$ in stable failures semantics. Algorithm 2 returns true only when working is empty, which implies that Dist(antichain) $>$ Dist(working) is not true. By invariant $2, \mathcal{L}_{1}$ refines $\mathcal{L}_{2}$ in stable failures semantics.

Example 2. If Algorithm 2 is applied to the example presented in Figure 1, the reduction remains the same as for trace refinement checking (from 7 states to 3 states). We show another example with some counterexamples, as shown in Figure 2. Notice that 
the refusal set of $s_{2}$ is $\{b\}$, and $s_{1}, s_{1}^{\prime}, s_{2}^{\prime}$ do not refuse any event. Then the two states with red circles are SFR-witness states. Since $\left\{s_{1}^{\prime}\right\} \subseteq\left\{s_{1}^{\prime}, s_{2}^{\prime}\right\}$, the search does not continue from the state $\left(s_{1},\left\{s_{1}^{\prime}, s_{2}^{\prime}\right\}\right)$. We can see that a SFR-witness state which is reachable from $\left(s_{1},\left\{s_{1}^{\prime}, s_{2}^{\prime}\right\}\right)$ is also reachable from $\left(s_{1},\left\{s_{1}^{\prime}\right\}\right)$ after the pruning of states. In this case, Algorithm 2 generates 3 states which are labelled with Anti-Chain, while the classical algorithm may generate 4 states before finding a SFR-witness state. Moreover, Algorithm 2 may find a shorter witness trace than the classical algorithm.

\subsection{Failures-Divergence Refinement Checking}

In the following, we show how to adopt anti-chain for failures-divergence refinement checking. Let $\mathcal{L}=(S$, init, Act, $T)$ be an LTS. Given a state $s, s$ diverges if and only if $s$ can performance an infinite number of $\tau$-transitions. A trace $t r$ is divergent, written as $\operatorname{div}(t r)$, if and only if there exists a prefix pre of $t r$ or $t r$ itself such that init $\stackrel{p r e}{\rightsquigarrow} s$ and $s$ diverges. We write divergences $(\mathcal{L})$ to be $\{t r \mid \operatorname{div}(\operatorname{tr})\}$.

Definition 5 (Failures-Divergence Refinement). Let $\mathcal{L}_{i}$ where $i \in\{1,2\}$ be two LTSs. $\mathcal{L}_{1}$ refines $\mathcal{L}_{2}$ in failures-divergence semantics if and only if divergences $\left(\mathcal{L}_{1}\right) \subseteq$ divergences $\left(\mathcal{L}_{2}\right)$ and failures $\left(\mathcal{L}_{1}\right) \subseteq$ failures $\left(\mathcal{L}_{2}\right)$.

In the following, we extend Algorithm 2 for failures-divergence refinement checking. The algorithm is shown in Algorithm 3. Given a set of state $x$, we say that $x$ diverges if there exists $s \in x$ such that $s$ diverges. Like in the existing failures-divergence refinement checking algorithm [1], the idea is to search for a FDR-witness state $(x, y)$ of $\mathcal{L}_{1} \times \operatorname{det}\left(\mathcal{L}_{2}\right)$ such that $y=\varnothing$ or refusals $(x) \nsubseteq$ refusals $(y)$ or $x$ diverges but not $y$.

Lemma 2. For every state $\left(s_{1}, s_{2}\right)$ of $\mathcal{L}_{1} \times \operatorname{det}\left(\mathcal{L}_{2}\right)$, for all $\left(s_{1}, s_{2}^{\prime}\right)$ in the product, if $s_{2}^{\prime} \subseteq s_{2}$, then a FDR-witness state is reachable from $\left(s_{1}, s_{2}\right)$ implies a FDR-witness state is reachable from $\left(s_{1}, s_{2}^{\prime}\right)$.

Proof By induction. The base case is that $\left(s_{1}, s_{2}\right)$ is a FDR-witness state, then $s_{2}=\varnothing$ or refusals $\left(s_{1}\right) \nsubseteq$ refusals $\left(s_{2}\right)$ or $s_{1}$ diverges and $s_{2}$ does not. Because $s_{2}^{\prime} \subseteq s_{2}$ by assumption, refusals $\left(s_{2}^{\prime}\right) \subseteq$ refusals $\left(s_{2}\right)$ and if $s_{2}^{\prime}$ diverges, so does $s_{2}$. Thus, $\left(s_{1}, s_{2}^{\prime}\right)$ is a SFR-witness state since $s_{2}=\varnothing$ implies $s_{2}^{\prime}=\varnothing$; refusals $\left(s_{1}\right) \not \subset$ refusals $\left(s_{2}\right)$ implies refusals $\left(s_{1}\right) \nsubseteq$ refusals $\left(s_{2}^{\prime}\right)$; and $s_{2}$ not divergent implies that $s_{2}^{\prime}$ does not diverge either. Next, we prove the induction step. Assume that $\left(s_{1}, s_{2}\right)$ satisfies the condition. Let $(x, y)$ be a state of the product such that $(x, y) \stackrel{e}{\rightarrow}\left(s_{1}, s_{2}\right)$. For all $\left(x, y^{\prime}\right)$ such that $y^{\prime} \subseteq y$, we can get $\left(x, y^{\prime}\right) \stackrel{e}{\rightarrow}\left(s_{1}, s_{2}^{\prime}\right)$ such that $s_{2}^{\prime} \subseteq s_{2}$. Therefore, the induction step holds by induction hypothesis. Thus, the lemma is true.

Theorem 3. Algorithm 3 returns true if and only if divergences $\left(\mathcal{L}_{1}\right) \subseteq$ divergences $\left(\mathcal{L}_{2}\right)$ and failures $\left(\mathcal{L}_{1}\right) \subseteq$ failures $\left(\mathcal{L}_{2}\right)$.

Proof Define $\operatorname{Dist}(S) \in N \cup\{\infty\}$ as the length of the shortest FDR-witness trace from a state $S$ of $\mathcal{L}_{1} \times \operatorname{det}\left(\mathcal{L}_{2}\right)$ (if a FDR-witness state is not reachable from $S, \operatorname{Dist}(S)=$ $\infty)$. Given a set of states States, if States $=\varnothing$, Dist (States $)=\infty$, otherwise, $\operatorname{Dist}($ States $)=\min _{S \in \text { States }} \operatorname{Dist}(S)$. The predicate FDR(States) is true if and only if all the states in States are not FDR-witness states. The correctness of Algorithm 3 can be proved similarly as for Algorithm 2, using the following two invariants. 


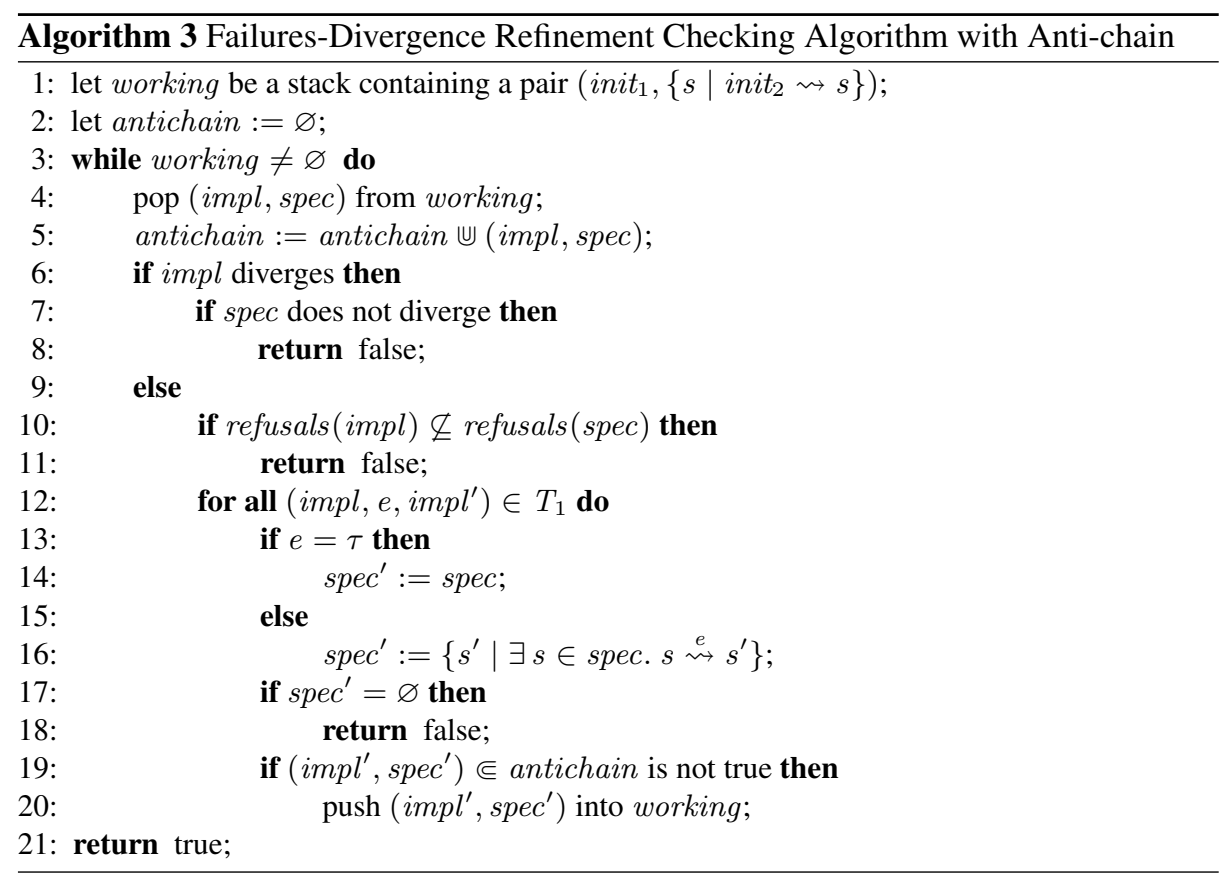

1. $\neg F D R($ antichain $\cup$ working $) \Rightarrow \neg F D R\left(\left\{\left(i,\left\{s \mid\right.\right.\right.\right.$ init $\left.\left._{2} \rightsquigarrow s\right\}\right) \mid i \in$ init $\left.\left._{1}\right\}\right)$.

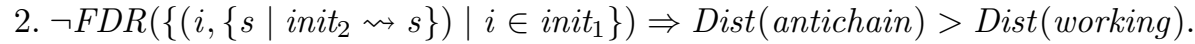

Example 3. We use the example shown in Figure 3 to demonstrate how algorithm 3 works. The state $s_{2}$ in LTS $\mathcal{L}_{1}$ has a self-loop labeled with $\tau$. The two states with red circles are FDR-witness states now. Since $\left\{s_{1}^{\prime}\right\} \subseteq\left\{s_{1}^{\prime}, s_{2}^{\prime}\right\}$, the search does not continue from the state $\left(s_{1},\left\{s_{1}^{\prime}, s_{2}^{\prime}\right\}\right)$. We can see that a FDR-witness state which is reachable from $\left(s_{1},\left\{s_{1}^{\prime}, s_{2}^{\prime}\right\}\right)$ is also reachable from $\left(s_{1},\left\{s_{1}^{\prime}\right\}\right)$ after pruning the states.

\subsection{Implementation and Evaluation}

The proposed algorithms have been adopted in the Process Analysis Toolkit (PAT) [13]. PAT is designed for systematic validation of distributed/concurrent systems using stateof-the-art model checking techniques. In the following, we evaluate the performance of the algorithms using a range of real-life parameterized systems. All the systems are embedded in the PAT package and available online. The data is obtained with Intel(R) Core(TM) i7-2640M CPU at 2.80GHz and 8GB RAM.

The pairs of LTSs (one as implementation and one as specification) are generated from different systems or same systems with different parameters. The systems include a multi-valued register simulation system with one reader or multiple readers [4], an implementation of concurrent stack with or without linearization point [15], a mailbox system [3], a system of scalable nonzero indicator [9] and the dining philosopher 


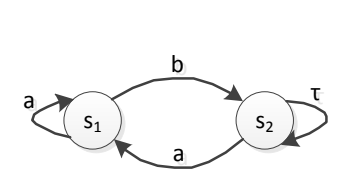

(a) LTS $\mathcal{L}_{1}$

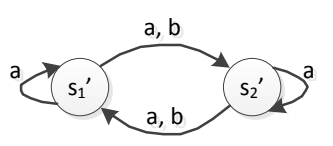

(b) LTS $\mathcal{L}_{2}$

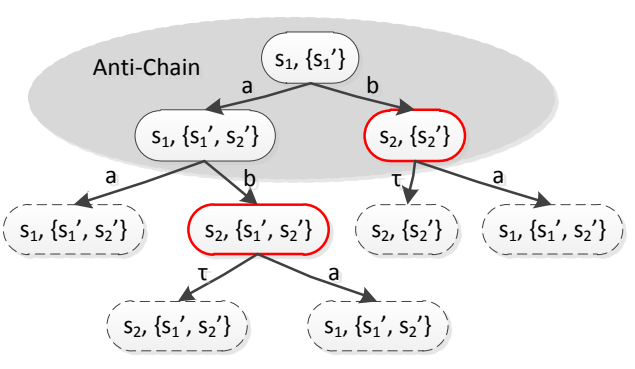

(c) a run of Algorithm 3 while checking failures $\left(\mathcal{L}_{1}\right) \subseteq$ failures $\left(\mathcal{L}_{2}\right)$ and divergences $\left(\mathcal{L}_{1}\right) \subseteq \operatorname{divergences}\left(\mathcal{L}_{2}\right)$

Fig. 3. Failures-Divergence Refinement Checking Algorithm with Anti-Chain
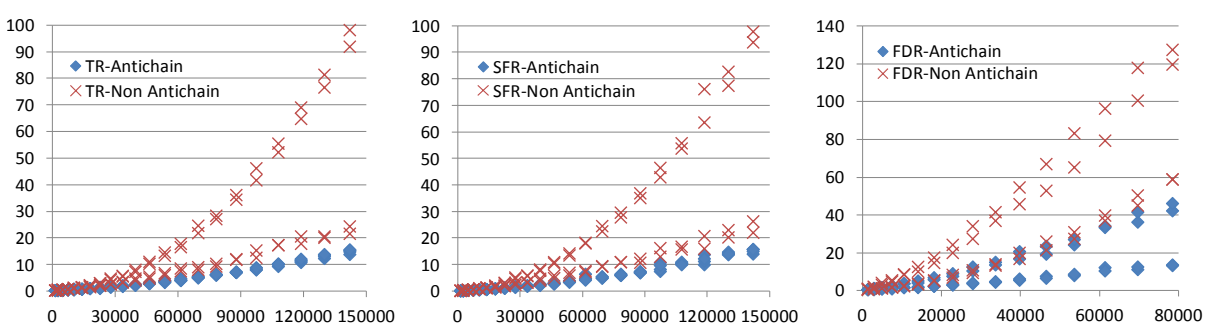

Fig. 4. Refinement Checking Results of Concurrent Stack Implementation

problem [11]. In total about 300 pairs of LTS were generated to compare the anti-chain algorithms and the classical ones for all three kinds of refinement checking.

Figure 4 shows the statistics of a typical example, i.e., the concurrent stack, from which we can see significant performance improvement. In the figure, the horizontal axis is the sum of the sizes of the two LTSs for refinement checking, and the vertical axis is the execution time (in seconds) of the corresponding algorithm. Each point shows the checking time for a pair of LTSs. We can see that for all three kinds of refinement checking, anti-chain based algorithms offer significantly better performance. The complete experimental results, with the refinement checking assertions always being valid, are summarized in Table 1. Notice that if the pair of LTSs are equivalent in terms of traces or failures or failures-divergence, we can perform the refinement checking in both directions. This is shown in the table using two columns $\subseteq$ and $\supseteq$. A few cases are marked as '_-' as the result is false, which are discussed later. Furthermore, 'unknown' means either out of memory or running for more than 30 minutes. It can be observed that the speedup differs for different systems. In most cases, the anti-chain approach has a much better performance than the classical one, e.g., in the concurrent stack linearization point implementation, it is 30.28 times faster for stable failures refinement checking and 12.16 times faster for failures-divergence refinement checking. Moreover, the larger the system is, the larger the speedup is. In some cases, anti-chain can not reduce the number of states at all simply because the specifications are deterministic. In some cases (e.g., SNZI), although anti-chain reduces the number of states, the benefit is not significant enough to overcome the computational overhead of the anti-chain operations defined in section 2.2. 


\begin{tabular}{|c|c|c|c|c|c|c|c|}
\hline \multirow{2}{*}{ System } & \multirow{2}{*}{ Size } & \multicolumn{2}{|c|}{$\begin{array}{c}\text { Trace } \\
\text { (Speedup) }\end{array}$} & \multicolumn{2}{|c|}{$\begin{array}{l}\text { Stable Failures } \\
\text { (Speedup) }\end{array}$} & \multicolumn{2}{|c|}{$\begin{array}{c}\begin{array}{c}\text { Failures-Divergence } \\
\text { (Speedup) }\end{array} \\
\end{array}$} \\
\hline & & $\subseteq$ & $\supseteq$ & $\subseteq$ & $\supseteq$ & $\subseteq$ & $\supseteq$ \\
\hline Multi-valued Register & $0-10000$ & 2.21 & 1.42 & 2.32 & 1.63 & 3.48 & 1.46 \\
\hline Simulation with & $10000-100000$ & 4.54 & 2.14 & 4.45 & 2.09 & 6.61 & 1.73 \\
\hline 1 Reader and 1 Writer & $100000-700000$ & 6.74 & 2.88 & 6.64 & 2.92 & unknown & 2.59 \\
\hline Multi-valued Register & $0-10000$ & 2.17 & 1.49 & 1.99 & 1.45 & 3.33 & 1.43 \\
\hline Simulation with & $10000-100000$ & 3.32 & 2.05 & 3.32 & 2.11 & 3.55 & 1.70 \\
\hline Multiple Readers & $100000-700000$ & 6.45 & 2.68 & 6.14 & 2.72 & unknown & 3.16 \\
\hline Concurrent & $0-10000$ & 1.48 & 1.85 & 1.63 & 1.72 & 2.26 & 1.60 \\
\hline Stack & $10000-100000$ & 1.70 & 3.72 & 1.71 & 3.71 & 2.71 & 3.22 \\
\hline Implementation & $100000-200000$ & 1.62 & 5.90 & 1.56 & 6.44 & 2.85 & 4.96 \\
\hline Concurrent Stack & $0-10000$ & 0.75 & 5.33 & - & 5.99 & - & 2.70 \\
\hline Linearization Point & $10000-30000$ & 0.84 & 13.94 & - & 14.11 & - & 5.00 \\
\hline Implementation & $30000-60000$ & 0.91 & 30.37 & - & 30.28 & - & 12.16 \\
\hline Mailbox & $0-700000$ & 1.13 & 1.54 & 1.11 & 1.61 & 1.39 & 1.01 \\
\hline SNZI & $0-50000$ & 0.89 & 2.45 & 0.93 & 2.42 & 1.03 & 1.14 \\
\hline Dining Philosopher & $0-50000$ & 0.99 & 1.14 & - & 1.02 & - & 1.17 \\
\hline
\end{tabular}

Table 1. Testing on Refinement Checking Assertions which are valid

\begin{tabular}{|c|c|c|c|c|c|c|c|}
\hline System & Size & $\begin{array}{c}\text { Trace } \\
\text { With AC(s) }\end{array}$ & $\begin{array}{c}\text { Trace } \\
\text { W/o AC(s) }\end{array}$ & $\begin{array}{c}\text { Stable Failures } \\
\text { With AC(s) }\end{array}$ & $\begin{array}{c}\text { Stable Failures } \\
\text { W/o AC(s) }\end{array}$ & $\begin{array}{c}\text { Failures-Divergence } \\
\text { With AC(s) }\end{array}$ & $\begin{array}{c}\text { Failures-Divergence } \\
\text { W/o AC(s) }\end{array}$ \\
\hline Multi-valued & 3175 & 0.10 & 0.42 & 0.09 & 0.43 & 0.44 & 2.88 \\
Register & 24655 & 1.52 & 12.12 & 1.71 & 12.02 & 9.78 & 146.95 \\
Simulation with & 117288 & 3.92 & 136.37 & 3.57 & 138.50 & 48.52 & 985.24 \\
Multiple Readers & 194455 & 22.11 & 294.71 & 21.51 & 299.21 & 180.91 & unknown \\
\hline
\end{tabular}

Table 2. Testing on Refinement Checking Assertions which are invalid

In presence of counterexamples, anti-chain based algorithms may find the counterexample more quickly. For instance, the verification results with the register example (with multiple readers), shown in Table 2 ('Anti-Chain' and 'Without' are denoted as $\mathrm{AC}$ and W/o for short), evidences that anti-chain finds the counterexample more quickly in all three kinds of refinement checking. Nonetheless, we remark that because the algorithms are on-the-fly, whether the counterexample is found earlier depends on the searching order and therefore sometime anti-chain based algorithms may be slower if a 'wrong' order is taken.

\section{Probabilistic Refinement Checking with Anti-Chain}

In this section, we show that anti-chain can be used to improve a particular kind of probabilistic refinement checking, i.e., the implementation is given as an MDP and the specification is given as an NFA.

\subsection{MDP and Probabilistic Model Checking}

Given a set of states $S$, a distribution is a function $\mu: S \rightarrow[0,1]$ such that $\Sigma_{s \in S} \mu(s)=$ 1. Let $\operatorname{Distr}(S)$ be the set of all distributions over $S$. A Markov Chain is a tuple $\mathcal{M}=$ ( $S$, init, Act, $\operatorname{Pr}$ ) where $S$ is a countable set of states; init $\in S$ is an initial state; Act is a set of events; and $\operatorname{Pr}: S \times A c t \times S \rightarrow[0,1]$ is a labeled transition probability function such that for all state $s \in S, \Sigma_{s^{\prime} \in S} \operatorname{Pr}\left(s, e, s^{\prime}\right)=1$. Notice that Markov Chains are deterministic as there is only one event (and one distribution) at each state. 
A sequence of alternating states and events $\pi=\left\langle s_{0}, e_{0}, s_{1}, e_{1}, \cdots, e_{n}, s_{n+1}\right\rangle$ is a path of $\mathcal{M}$ if $\operatorname{Pr}\left(s_{i}, e_{i}, s_{i+1}\right)>0$ for all $i$. The probability of executing $\pi$ from $s_{0}$, written as $\operatorname{Pr}(\mathcal{M}, \pi)$, is $\operatorname{Pr}\left(s_{0}, e_{0}, s_{1}\right) \times \operatorname{Pr}\left(s_{1}, e_{1}, s_{2}\right) \times \cdots \times \operatorname{Pr}\left(s_{n}, e_{n}, s_{n+1}\right)$. It is often also interesting to find out the probability of reaching a certain set of states (e.g., what is the probability of reaching the state of system failure?). Given a set of target states $G$, the probability of reaching any state in $G$ from a starting state $s_{0}$, written as $\operatorname{Pr}\left(\mathcal{M}, s_{0}, G\right)$, is the accumulated probability of all paths from $s_{0}$ to any state in $G$, which can be calculated systematically [5]. Given a path $\pi$, we define $\operatorname{trace}(\pi)$ to be the sequence of visible events in $\pi$. We write $\operatorname{Pr}\left(\mathcal{M}, s_{0}, t r\right)$ to denote the probability of exhibiting a trace $t r$ from state $s_{0}$, which is the accumulated probability of all paths $\pi$ from $s_{0}$ such that $\operatorname{trace}(\pi)=\operatorname{tr}$. Given a set of traces $\operatorname{Tr}$, the probability of $\mathcal{M}$ exhibiting any trace in $\operatorname{Tr}$ from state $s_{0}$ is the accumulated probability $\Sigma_{t r \in \operatorname{Tr}} \operatorname{Pr}\left(\mathcal{M}, s_{0}, t r\right)$.

Different from Markov Chains, an MDP can express both probabilistic choices and non-determinism. An MDP is a tuple $\mathcal{D}=(S$, init, $A c t, P r)$ where $S$ is a set of system states; init $\in S$ is the initial system configuration ${ }^{5}$; Act is a set of actions; $\operatorname{Pr}: S \times$ $A c t \times \operatorname{Distr}(S)$ is a transition probability function such that for all states $s \in S$ and $a \in$ Act: $\Sigma_{s^{\prime} \in S} \operatorname{Pr}\left(s, a, s^{\prime}\right) \in\{0,1\}$. Notice that there could be multiple events at any state. A transition of the system is written as $s \stackrel{e}{\rightarrow} \mu$ where $\mu$ is a distribution. A path of $\mathcal{M}$ is a sequence of alternating states, events and distributions $\pi=\left\langle s_{0}, e_{0}, \mu_{0}, s_{1}, e_{1}, \mu_{1}, \cdots\right\rangle$ such that $s_{0}=i n i t$ and $s_{i} \stackrel{e_{i}}{\rightarrow} \mu_{i}$ and $\mu_{i}\left(s_{i+1}\right)>0$ for all $i$. Given a path $\pi$, we define $\operatorname{trace}(\pi)$ to be the sequence of visible events in $\pi$.

Intuitively speaking, given a system configuration, firstly an event and a distribution is selected non-deterministically by the scheduler, and then one of successor states is reached according to the probability distribution. A scheduler is a function deciding which event and distribution to choose based on the execution history. With a scheduler $\delta$, we effectively obtain a Markov Chain from $\mathcal{D}$, written as $\mathcal{D}_{\delta}$. Note that with different scheduling, the probability of reaching a state or exhibiting a trace may be different. The measurement of interest is thus the maximum and minimum probability. Given a set of target states $G$ and an MDP $\mathcal{D}$, the maximum probability of reaching any state in $G$ from state $s_{0}$ is defined as $\mathcal{P}_{\max }\left(\mathcal{D}, s_{0}, G\right)=\sup _{\delta} \operatorname{Pr}\left(\mathcal{D}_{\delta}, s_{0}, G\right)$. Note that the supremum ranges over all, potentially infinitely many, schedulers. Accordingly, the minimum is written as $\mathcal{P}_{\text {min }}\left(\mathcal{D}, s_{0}, G\right)$. Similarly, we define the maximum probability of exhibiting a trace in a set $\operatorname{Tr}$ by $\mathcal{D}$ from $s_{0}$.

$$
\mathcal{P}_{\text {max }}\left(\mathcal{D}, s_{0}, \operatorname{Tr}\right)=\sup _{\delta}\left(\Sigma_{t r \in \operatorname{Tr}} \operatorname{Pr}\left(\mathcal{D}_{\delta}, s_{0}, t r\right)\right)
$$

Accordingly, the minimum is written as $\mathcal{P}_{\min }\left(\mathcal{D}_{\delta}, s_{0}, \operatorname{Tr}\right)$.

Example 4. The following shows a simple example MDP.

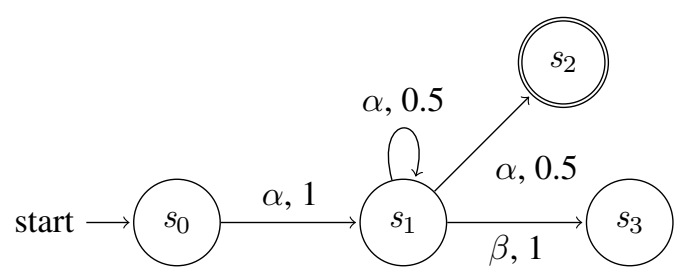

\footnotetext{
${ }^{5}$ This is a simplified definition. In general, there can be an initial distribution.
} 
where $s_{0}$ is the initial state and $s_{2}$ is a target state. For simplicity, we omit the selfloop of $s_{2}$ and $s_{3} . s_{1}$ has two distributions, following two actions $\alpha$ and $\beta$. If $s_{1}$ nondeterministically chooses $\alpha$, then it has equal probability to transfer to $s_{2}$ or stay in $s_{1}$; and if $\beta$ is chosen, it will transfer to $s_{3}$ with probability 1 .

Definition 6 (Refinement Probability). Let $\mathcal{D}=(S$, init, Act, $\operatorname{Pr})$ be an $M D P ; \mathcal{L}$ be an LTS. The maximum probability of $\mathcal{D}$ trace-refining $\mathcal{L}$ is $\mathcal{P}_{\text {max }}(\mathcal{D}$, init, traces $(\mathcal{L}))$. The minimum is $\mathcal{P}_{\text {min }}(\mathcal{D}$, init, traces $(\mathcal{L}))$.

Intuitively, the probability of $\mathcal{D}$ refines $\mathcal{L}$ is the probability of $\mathcal{D}$ exhibiting a trace of $\mathcal{L}$. As we mention earlier, the probability may vary due to different scheduling.

Definition 7 (MDP and LTS Synchronous Product). Let $\mathcal{D}=\left(S_{d}\right.$, init $\left._{d}, A c t_{d}, P r_{d}\right)$ be an MDP; $\mathcal{L}=\left(S_{l}\right.$, init $\left._{l}, A c t_{l}, T\right)$ be an LTS such that $\tau \notin A c t_{l}$. The synchronous product of $\mathcal{D}$ and $\mathcal{L}$, written as $\mathcal{D} \times \mathcal{L}$, is an $M D P(S$, init, Act, $\operatorname{Pr})$ such that $S=$ $S_{d} \times S_{l} ;$ init $=\left(\right.$ init $_{d}$, init $\left._{l}\right) ; A c t=A c t_{d} \cup A c t_{l}$; and Pr is defined as follows.

- If $\left(s_{1}, \tau, \mu\right) \in \operatorname{Pr}_{d},\left(\left(s_{1}, s_{2}\right), \tau, \mu^{\prime}\right) \in \operatorname{Pr}$ for all $s_{2} \in S_{l}$ such that for all $s_{1}^{\prime} \in S_{d}$, $\mu^{\prime}\left(\left(s_{1}^{\prime}, s_{2}\right)\right)=\mu\left(s_{1}^{\prime}\right)$;

- If $\left(s_{1}, e, \mu\right) \in \operatorname{Pr}_{d}$ and $\left(s_{2}, e, s_{2}^{\prime}\right) \in T_{2},\left(\left(s_{1}, s_{2}\right), e, \mu^{\prime}\right) \in \operatorname{Pr}$ such that for all $s_{1}^{\prime} \in S_{d}, \mu^{\prime}\left(\left(s_{1}^{\prime}, s_{2}^{\prime}\right)\right)=\mu\left(s_{1}^{\prime}\right)$.

A state $\left(s_{1}, s_{2}\right)$ of the product $\mathcal{D} \times \mathcal{L}$ is a TR-witness state if and only if $s_{2}=\varnothing$. In [14], we show that the refinement probability can be calculated systematically by (1) building the deterministic LTS $\operatorname{det}(\mathcal{L}) ;(2)$ computing the synchronous product of $\mathcal{D}$ and $\operatorname{det}(\mathcal{L})$; $3)$ calculating the maximum/minimum probability of reaching any TR-witness state.

Theorem 4. Let $\mathcal{D}=\left(S_{d}\right.$, init $\left._{d}, A c t_{d}, P r_{d}\right)$ be an $M D P ; \mathcal{L}=\left(S_{l}\right.$, init $_{l}$, Act,$\left.T\right)$. Let $G$ be the set of TR-witness states of $\mathcal{D} \times \operatorname{det}(\mathcal{L})$.

$-\mathcal{P}_{\text {max }}\left(\mathcal{D}\right.$, init $\left._{d}, \operatorname{traces}(\mathcal{L})\right)=\mathcal{P}_{\max }\left(\mathcal{D} \times \operatorname{det}(\mathcal{L}),\left(\right.\right.$ init $_{d}$, init $\left.\left._{l}\right), G\right)$

- $\mathcal{P}_{\text {min }}(\mathcal{D}$, traces $(\mathcal{L}))=\mathcal{P}_{\text {min }}\left(\mathcal{D} \times \operatorname{det}(\mathcal{L}),\left(\right.\right.$ init $_{d}$, init $\left.\left._{l}\right), G\right)$

Based on the above theorem, the probabilistic refinement checking problem is reduced to a probabilistic reachability problem, which can be solved by two standard methods. One is by solving a linear program. That is, we firstly associate a variable $x_{s}$ to each state $s$ in $\mathcal{P}$ to represent the probability of reaching any target state from $s$; then we construct a linear program which constraints the value of every $x_{s}$ using a set of linear inequalities, based on the probability transition function; and lastly, we solve the linear program to get the maximum/minimum value of each $x_{s}$. Notice that the solution of state $\left(\right.$ init $_{d}$, init $\left._{l}\right)$ is the refinement probability. The other is to iteratively approximate the probability through graph traversing. Notice that for systems having large state space, it is impractical to store the entire linear program and solve it directly, therefore the iterative calculation approach is more widely used in probabilistic verification. As a result, in this paper we just focus on this approach.

Example 5. In the following, we show how the iterative calculation method works using the simple example shown in Example 4. That is, the maximal probability from initial state $s_{0}$ to accepting state $s_{2}$ is calculated step by step. Assume $p_{i}^{k}$ is the maximal 
probability of $s_{i}$ after the $k$-th iteration. Starting from the target state $s_{2}$, in $k$-th iteration we update the probability of states which could reach $s_{2}$ in exact $k$ steps. Obviously, $p_{0}^{0}=p_{1}^{0}=0$. As $p_{2}^{k}=1$ and $p_{3}^{k}=0$ for any $k, k$ is ignored in these two states. In the 1st iteration, only $p_{1}$ can be updated, and $p_{1}^{1}=\max \left\{0.5 \times p_{1}^{0}+0.5 \times p_{2}, 1 \times p_{3}\right\}=$ $\max \{0.5,0\}=0.5$.; in the 2nd iteration, both $p_{0}$ and $p_{1}$ can be updated. It is trivial to show $p_{0}^{2}=p_{1}^{1}=0.5$, and $p_{1}^{2}=\max \left\{0.5 \times p_{1}^{1}+0.5 \times p_{2}, 1 \times p_{3}\right\}=\max \{0.75,0\}=0.75$. Iteratively, $p_{0}$ and $p_{1}$ in the long run can be calculated. A user-defined threshold is usually necessary to terminate the calculation, according to the desired precision.

\subsection{Anti-chain Based Approach}

Now we introduce how anti-chain can be used to speed up the iterative calculation approach, by first introducing a lemma.

Lemma 3. Let $\mathcal{D}=\left(S_{d}\right.$, init $\left._{d}, A c t_{d}, P r_{d}\right)$ be an MDP; $\mathcal{L}=\left(S_{l}\right.$, init $\left._{l}, A c t_{l}, T\right)$. Let $\mathcal{P}$ be $\mathcal{D} \times \operatorname{det}(\mathcal{L})$. Let $G$ be the set of TR-witness states of $\mathcal{P}$. For all state $\left(u_{1}, v_{1}\right)$ and $\left(u_{2}, v_{2}\right)$ of $\mathcal{P}$ s.t. $\left(u_{2}, v_{2}\right) \leq\left(u_{1}, v_{1}\right), P r_{\max }\left(\mathcal{P},\left(u_{1}, v_{1}\right), G\right) \geq P r_{\max }\left(\mathcal{P},\left(u_{2}, v_{2}\right), G\right)$ and $\operatorname{Pr}_{\text {min }}\left(\mathcal{P},\left(u_{1}, v_{1}\right), G\right) \geq \operatorname{Pr}_{\text {min }}\left(\mathcal{P},\left(u_{2}, v_{2}\right), G\right)$.

Proof The above can be proved with an induction. The base case is that $\left(u_{2}, v_{2}\right)$ is in $G$. By definition, $\left(u_{1}, v_{1}\right)$ must be in $G$ and therefore the lemma holds. Next, we show the induction step. Assume that $\left(u_{2}^{\prime}, v_{2}^{\prime}\right)$ satisfies the lemma above. For every distribution $\mu_{2}$ from $\left(u_{2}, v_{2}\right)$, by Definition 7, there must exist a distribution $\mu_{1}$ from $\left(u_{1}, v_{1}\right)$ and for every state $\left(u_{2}^{\prime}, v_{2}^{\prime}\right)$, there exists $\left(u_{1}^{\prime}, v_{1}^{\prime}\right)$ such that $\mu_{2}\left(\left(u_{2}^{\prime}, v_{2}^{\prime}\right)\right)=\mu_{1}\left(\left(u_{1}^{\prime}, v_{1}^{\prime}\right)\right)$ and $\left(u_{2}^{\prime}, v_{2}^{\prime}\right) \leq\left(u_{1}^{\prime}, v_{1}^{\prime}\right)$. By induction hypothesis, we have $\operatorname{Pr}_{\max }\left(\mathcal{P},\left(u_{1}^{\prime}, v_{1}^{\prime}\right), G\right) \geq$ $\operatorname{Pr}_{\text {max }}\left(\mathcal{P},\left(u_{2}^{\prime}, v_{2}^{\prime}\right), G\right)$ and $\operatorname{Pr}_{\text {min }}\left(\mathcal{P},\left(u_{1}^{\prime}, v_{1}^{\prime}\right), G\right) \geq \operatorname{Pr}_{\text {min }}\left(\mathcal{P},\left(u_{2}^{\prime}, v_{2}^{\prime}\right), G\right)$. Thus we have $\operatorname{Pr}_{\text {max }}\left(\mathcal{P},\left(u_{1}, v_{1}\right), G\right) \geq \operatorname{Pr}_{\max }\left(\mathcal{P},\left(u_{2}, v_{2}\right), G\right)$ and $\operatorname{Pr}_{\min }\left(\mathcal{P},\left(u_{1}, v_{1}\right), G\right) \geq$ $P r_{\min }\left(\mathcal{P},\left(u_{2}, v_{2}\right), G\right)$. Therefore, we conclude that the lemma holds.

Compared to probabilistic reachability calculation for a general MDP, the above lemma gives us additional information, which can be potentially useful in speeding up the calculation. In the following, we discuss how we can make use of the information so as to improve the probabilistic refinement checking using the iterative calculation method.

The first step is building the product MDP meanwhile finding the target states, which is shown in Algorithm 4. The implementation and specification are defined in Definition 7. Different from the non-probabilistic cases, the state space cannot be reduced in the probabilistic models; instead, we define a function $s u b$ of the product state $s$ satisfying $s . s u b=\{t \mid t \in S \wedge s \leq t\}$, where $S$ is the state space of the product MDP. Then the refinement checking is reduced to probabilistic reachability of a set of target states, denoted by Target. During the iterative calculation, whenever the probability of state $s$ is updated, e.g., to $p$, according to lemma 3, all states in $s . s u b$ whose probability is less than $p$ could be set to $p$ directly. This could speed up each iteration and potentially improve probabilistic refinement checking.

Now we evaluate whether the above method is indeed beneficial. The proposed probabilistic refinement checking algorithm has also been implemented in PAT. We evaluate it using a modified system based on the implementation of a distributed concurrent stack example [15]. Probabilistic choices are used to model a concurrent stack 


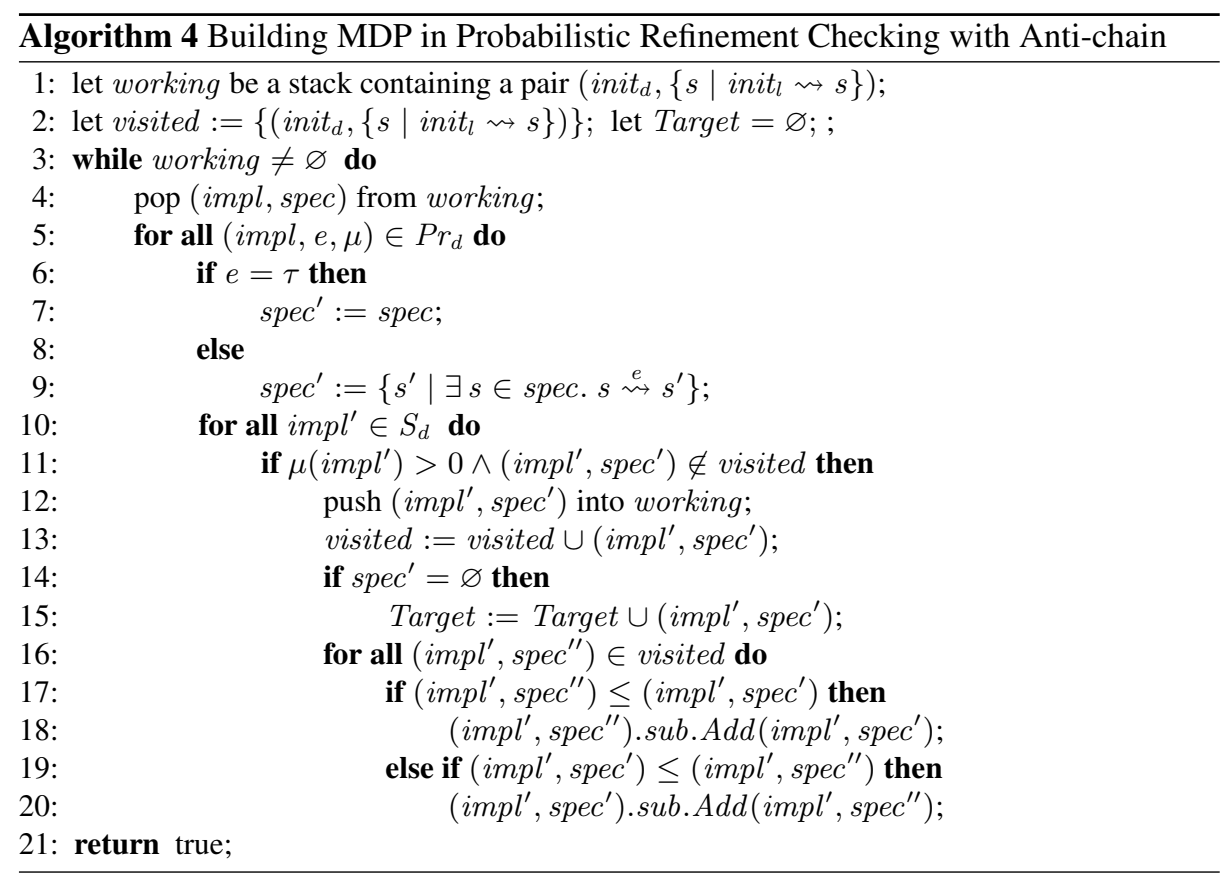

\begin{tabular}{|c|c|c|c|c|c|c|c|}
\hline \multirow{2}{*}{ System } & \multirow{2}{*}{ Size } & \multicolumn{3}{|c|}{ Verification Time (s) } & \multicolumn{3}{|c|}{ \#tates Involved in Iterations } \\
\cline { 3 - 8 } & & W/o AC & With AC & Gain & W/o AC & With AC & Gain \\
\hline $\mathrm{K}=2$ & 20600 & 2.74 & 2.21 & $19.3 \%$ & $4.2 \mathrm{M}$ & $3 \mathrm{M}$ & $28.6 \%$ \\
\hline $\mathrm{K}=3$ & 45584 & 15.98 & 12.04 & $24.6 \%$ & $18.6 \mathrm{M}$ & $11.7 \mathrm{M}$ & $37.1 \%$ \\
\hline $\mathrm{K}=4$ & 86704 & 48.72 & 37.50 & $22.6 \%$ & $55.5 \mathrm{M}$ & $36.2 \mathrm{M}$ & $34.8 \%$ \\
\hline $\mathrm{K}=5$ & 117408 & 123.9 & 80.83 & $34.9 \%$ & $130.7 \mathrm{M}$ & $76.3 \mathrm{M}$ & $41.6 \%$ \\
\hline $\mathrm{K}=6$ & 231440 & 271.2 & 182.6 & $32.7 \%$ & $272.1 \mathrm{M}$ & $160.7 \mathrm{M}$ & $40.9 \%$ \\
\hline $\mathrm{K}=7$ & 342544 & 511.1 & 340.3 & $33.5 \%$ & $515.2 \mathrm{M}$ & $298.8 \mathrm{M}$ & $42.0 \%$ \\
\hline
\end{tabular}

Table 3. Experiments: Probabilistic Concurrent Stack Implementation

model composed by two processes, so as to capture the situation in which the communication between different processes fails from time to time. Failures do exist in real world cases and the experiments results are summarized in Table 3.

We compare the efficiency of the implementation with and without (W/o) Antichain (AC) using several cases. $K$ means length of the stack; Size indicates the number of states in the whole system; \#States Involved in Iterations represents the total number of states involved in the iterative calculation. For example, a state $s$ updates its probability in two iterations, then \#States should increase two. From the experiments, we can see that the anti-chain approach could reduce the total number of states accumulated during the calculation, through dynamically updating states' probability based on the subset relation sub. This speeds up the verification around 29\%. We remark that the gains here are not as significant as the non-probabilistic cases, because the state space cannot be reduced; however, in some cases, it does shorten the verification time. 


\section{Conclusion}

In this work, we proposed to adopt anti-chain approach to improve stable failures refinement, failures-divergence refinement and probabilistic refinement checking. These algorithms have been implemented in model checking framework PAT, and some experiments based on benchmark systems demonstrated the dramatic improvement of the verification efficiency of our method. To our best of knowledge, we are the first to investigate anti-chain approaches for these refinement checking.

As for future work, we are trying to extend anti-chain based refinement checking approach in real-time system; meanwhile, we are exploring the refinement relation between probabilistic models, which may also benefit from anti-chain based method.

\section{References}

1. A. W. Roscoe. Model-checking CSP, chapter 21. Prentice-Hall, 1994.

2. P. A. Abdulla, Y.-F. Chen, L. Holk, R. Mayr, and T. Vojnar. When simulation meets antichains. In TACAS, volume 6015 of Lecture Notes in Computer Science, pages 158-174. Springer, 2010.

3. M. Aguilera, E. Gafni, and L. Lamport. The mailbox problem. Distributed Computing, 2008.

4. H. Attiya and J. Welch. Distributed Computing: Fundamentals, Simulations, and Advanced Topics, 2nd Edition. The Oxford University Press, 2004.

5. C. Baier and J. Katoen. Principles of Model Checking. The MIT Press, 2008.

6. A. Bouajjani, P. Habermehl, L. Holk, T. Touili, and T. Vojnar. Antichain-based universality and inclusion testing over nondeterministic finite tree automata. In CIAA, volume 5148 of Lecture Notes in Computer Science, pages 57-67. Springer, 2008.

7. K. Chatterjee, L. Doyen, T. A. Henzinger, and J.-F. Raskin. Algorithms for omega-regular games with imperfect information. In CSL, volume 4207 of Lecture Notes in Computer Science, pages 287-302. Springer, 2006.

8. L. Doyen and J. F. Raskin. Antichains for the automata-based approach to model checking. Logical Methods in Computer Science, 5(1:5):1-20, 2009.

9. F. Ellen, Y. Lev, V. Luchangco, and M. Moir. SNZI: Scalable nonzero indicators. In PODC, pages 13-22. ACM, 2007.

10. E. Filiot, N. Jin, and J.-F. Raskin. An antichain algorithm for ltl realizability. In $C A V$, volume 5643 of Lecture Notes in Computer Science, pages 263-277. Springer, 2009.

11. C. Hoare. Communicating Sequential Processes. Prentice-Hall, 1985.

12. A. W. Roscoe. On the expressive power of CSP refinement. Formal Aspects of Computing, 17(2):93-112, 2005.

13. J. Sun, Y. Liu, J. S. Dong, and J. Pang. PAT: Towards flexible verification under fairness. In $C A V$, volume 5643 of Lecture Notes in Computer Science, pages 709-714. Springer, 2009.

14. J. Sun, S. Song, and Y. Liu. Model checking hierarchical probabilistic systems. In ICFEM, volume 6447 of Lecture Notes in Computer Science, pages 388-403. Springer, 2010.

15. R. K. Treiber. Systems programming: Coping with parallelism. Technical report, IBM Almaden Research Center, 1986.

16. M. D. Wulf, L. Doyen, T. A. Henzinger, and J.-F. Raskin. Antichains: A new algorithm for checking universality of finite automata. In $C A V$, volume 4144 of Lecture Notes in Computer Science, pages 17-30. Springer, 2006.

17. M. D. Wulf, L. Doyen, N. Maquet, and J.-F. Raskin. Antichains: Alternative algorithms for 1tl satisfiability and model-checking. In TACAS, volume 4963 of Lecture Notes in Computer Science, pages 63-77. Springer, 2008. 